\title{
gonflement d'un sol contenant des sulfates et traité à la chaux et au ciment
}

\section{foundation heave on a lime and cement treated soil containing gypsum}

\author{
P. HABIB \\ Laboratoire de Mécanique des solides* \\ D. AVERSENC \\ SOPENA ingénierie géotechnique **
}

\section{Résumé}

Le cas du soulèvement anormal du dallage d'un bâtiment industriel dans la région parisienne est décrit; son origine est liée à la présence de gypse dans un remblai traité à la chaux et au ciment.

\section{Abstract \\ The anormal heave of the slab of an industrial building in the Paris area is descri- bed. The origin of the swelling is the presence of gypsum in a fill treated with lime and cement.}




\section{INTRODUCTION}

Les progrès réalisés dans l'efficacité des matériels utilisés pour le traitement par additifs des remblais argileux sont tels, tant au point de vue des appareils destinés à mélanger le sol et les additifs que des engins de compactage, que même des sols très argileux peuvent être utilisés pour préparer des sols industriels : à la limite, sachant qu'on peut tout traiter à la chaux ou au ciment, on traite un peu n'importe 'quoi et on ne s'occupe plus guère de la nature des remblais. Malheureusement, la présence de sulfates dans certains sols peut engendrer des gonflements et provoquer des accidents. Ces sulfates sont présents généralement sous forme de gypse ou de plâtre (remblai contenant des matériaux de démolition). On a pu effectivement en constater quelques-uns en région parisienne au cours des dernières années. Le cas décrit ici en est une illustration.

\section{DESCRIPTION DE L'OUVRAGE}

Pour augmenter la capacité de ses entrepôts à Louvres, petit village situé au nord de Paris près de l'aéroport de Roissy-en-France, une importante société a décidé la construction d'un nouveau bâtiment industriel adjacent et de niveau à son premier entrepôt. L'extension du bâtiment s'est faite en direction de l'ancienne carrière d'une briquetterie qui avait exploité du limon sur $7 \mathrm{~m}$ d'épaisseur ; cette carrière avait été partiellement comblée depuis longtemps. Pour réaliser la plate-forme du nouvel entrepôt, on a utilisé, comme remblais, des terres provenant de travaux de fouilles dont, à la suite d'une histoire longue et compliquée, il est actuellement impossible de savoir d'où elles proviennent. Il s'agit d'un sol marneux, contenant du limon ou d'un mélange de sols divers, contenant suffisamment d'argile, pour qu'il soit décidé de le traiter à la chaux et au ciment pour le rendre apte au compactage. Après nettoyage de la terre végétale, le fond de fouille a d'abord été traité à la chaux. Ensuite, les remblais d'apport ont été mis en place par couches successives ; ils ont été traités à la chaux, puis compactés sur une épaisseur de $1 \mathrm{~m}$, Enfin, les 0,30 à $0,40 \mathrm{~m}$ superficiels furent traités au ciment et compactés pour faire le fond de forme du dallage.

Le nouvel entrepôt est une construction légère et simple, constituée d'une charpente métallique, d'une couverture et d'un bardage, le tout étant porté par des pieux forés tubés de diamètre $0,60 \mathrm{~m}$, d'une longueur supérieure à $10 \mathrm{~m}$ pour trouver leur fiche dans les sables de Beauchamp, au-delà de l'ancien plancher de la carrière. En périphérie, les têtes de pieux sont reliées par une longrine de $0,20 \mathrm{~m} \times 0,80 \mathrm{~m}$, et les appuis, à l'intérieur du bâtiment, sont des appuis isolés. Les aléas du sous-sol justifient ce mode de fondation dont le comportement a été, dans l'ensemble excellent. Les poussées horizontales des portiques sont reprises en pieds, par des aciers noyés dans le dallage, l'effort horizontal étant transmis par frottement du dallage sur le sol. Le dallage repose directement sur le sol; il est dissocié du bâtiment, malheureusement pas assez comme on le verra plus loin. L'étude de sol faite en 1983 indiquait la possibilité d'un léger tassement des matériaux anciens du remblai de la carrière sous le poids du mètre et demi des remblais compactés nouveaux. Le sol industriel est constitué par de grandes dalles de $15 \mathrm{~cm}$ d'épaisseur, en béton armé d'un treillis soudé. Les joints sont sciés sur une profondeur de $4 \mathrm{~cm}$. L'emprise au sol est de $38 \mathrm{~m} \times 60 \mathrm{~m}$. Les produits entreposés sont extrêmement légers et non périssables; ils sont stockés en hauteur, en racks, ce qui nécessite un sol de roulage bien uni pour la circulation des engins de manutention et de levage.

La construction a été faite entre avril et aout 1983, par des entreprises sérieures et bien surveillées ; en particulier, l'efficacité du compactage des remblais a été contrôlée par des essais à la plaque, effectués par un laboratoire compétent et indépendant de l'entreprise; la réception de l'ensemble a été prononcée sans réserves.

\section{LES DÉSORDRES}

En avril 1984, quelques déformations différentielles du dallage ont été constatées; elles furent l'objet de raccords par mortier à la résine en aout 1984. Mais les désordres se sont amplifiés progressivement et consistaient en des défauts de planéité du dallage, associés à des fissures du béton (qu'on pouvait prendre pour des fissures de retrait), des désaffleurements des joints de structures des dalles de lordre du centimètre, et des cassures des joints donnant l'image d'un allongement du dallage. Les fissurations sur le dallage étaient « en étoiles n, au pourtour des points de gonflement les plus élevés. En périphérie, le muret situé sur la longrine a été soulevé (fig. 1 et 2). Le pied du bardage, fixé au muret, a été détérioré.

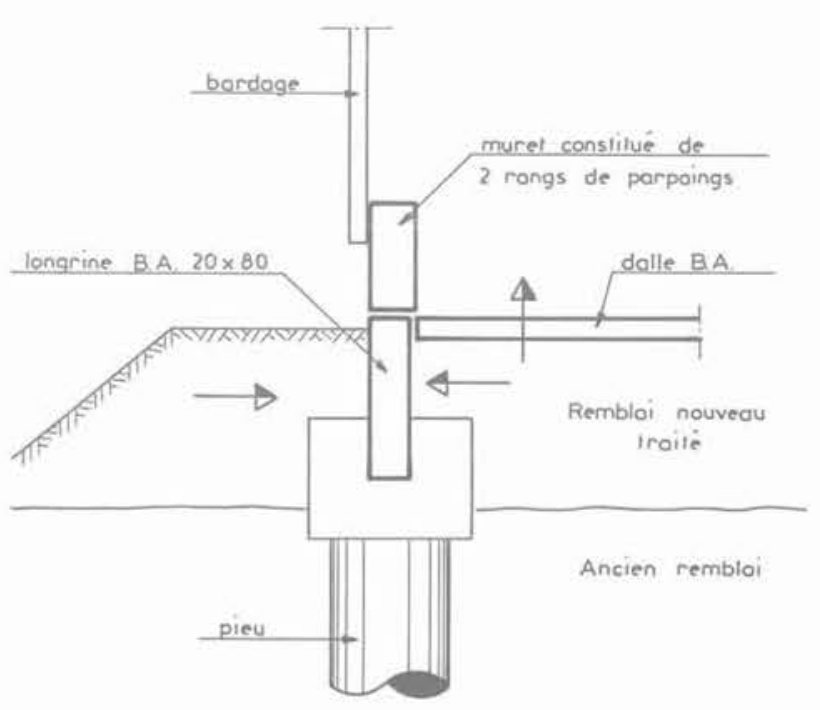

Fig. 1. - Avant gonflement.

Fig. 1. - Before swelling. 


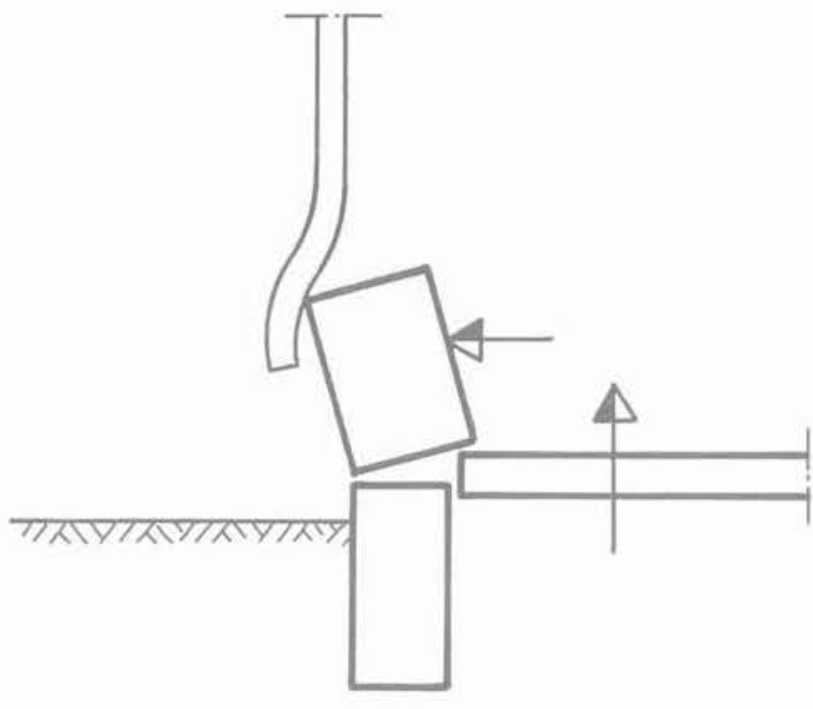

Fig. 2. - Après gonflement.

Fig. 2. - After swelling.

\section{ANALYSE DES CAUSES}

Les désordres pouvaient faire penser à un tassement des fondations sur pieux, alors que ceux-ci étaient nettement surabondants, ou à un gonflement du sol, ce qui était peu compréhensible puisque le sol était bien isolé de la pluie et que la nappe phréatique était très profonde (10 $\mathrm{m}$ environ).

La topographie montrait que les niveaux des massifs sur pieux correspondaient bien au niveau de construction, mais le niveau du sol était peut-être plus haut que prévu initialement, de quelques centimètres. Comme il s'agissait de l'ordre de grandeur de la précision du chantier, deux campagnes de topographie précise furent faites, à deux mois d'intervalle, en juillet et septembre 1985. Elles montrèrent une certaine dispersion des gonflements en fonction des emplacements des points de mesure dans le bâtiment, mais avec des maximums de $1 \mathrm{~mm} / \mathrm{mois}$, c'est-à-dire environ $1 \mathrm{~cm} / \mathrm{an}$. A peu près la moitié du bâtiment, soit $1000 \mathrm{~m}^{2}$, était affectée par des gonflements d'amplitudes diverses.

Pour déterminer la cause des gonflements, des sondages, avec prélèvements d'échantillons intacts et études en laboratoire, furent effectués. Les essais de laboratoire comportaient des essais de routine (teneur en eau, densité, identification), des essais de gonflement et des analyses chimiques.

Les essais de gonflement ont confirmé la bonne qualité du compactage avérée par les essais de réception. A la limite, on peut même dire que les remblais ont été un peu trop bien compactés, puisqu'il existait des endroits où la pression de gonflement était non négligeable et supérieure au poids des terres de $25 \mathrm{kPa}$, mais, à vrai dire, avec un potentiel de gonflement très petit puisque le gonflement libre de toute la couche aurait été de l'ordre de $1 \mathrm{~cm}$, ce qui est faible. De toutes façons, comme les sols n'étaient pas saturés et qu'il n'y avait pas eu de venues d'eau, le gonflement classique des sols argileux ne pouvait pas être à l'origine des faits constatés.

Il est évident, pour l'analyse chimique, que la recherche de la chaux des additifs est impossible dans un sol marneux, le calcium du calcaire étant indiscernable du calcium de la chaux, même si la couche traitée au ciment se distinguait par sa couleur grise de la couche traitée à la chaux. Les analyses, effectuées au C.E.B.T.P., ont donc comporté essentiellement la recherche du gypse et de l'ettringite. Le gypse a été trouvé, en quantités variables, dans tous les matériaux rapportés, anciens ou nouveaux. En reprenant les analyses faites lors des études préliminaires, on a même pu constater que, chaque fois que la recherche. du gypse avait été faite, on en avait trouvé, ce qui n'a absolument rien d'inattendu dans des sols à faible profondeur, dans cette partie de la région parisienne. De la même façon, de l'ettringite a été identifiée dans les remblais compactés du nouvel entrepôt, ce qui expliquait les gonflements en donnant l'origine chimique des désordres.

Le principe du traitement à la chaux d'un sol contenant de l'argile est le suivant :

- d'une part, l'apport de chaux vive diminue la teneur en eau naturelle et augmente la teneur en eau optimale, facilitant ainsi le compactage,

- d'autre part, les ions calcium se substituent aux ions échangeables des feuillets d'argile, rendant l'argile moins active. L'effet pratique sur le chantier est une désagrégation des mottes d'argile.

Le traitement mixte chaux et ciment permet d'obtenir des mélanges présentant une forte résistance mécanique et notamment une résistance à la traction de plusieurs centaines de kilopascals.

Dans ce traitement, l'apport de chaux conduit à la désagrégation du sol et permet donc de mélanger assez intimement le ciment. Une bonne répartition du ciment dans le sol est en effet indispensable à la réussite du traitement. Le ciment, lui, apporte l'essentiel de la résistance mécanique.

Ce traitement est classique dans la région parisienne depuis plus de trente ans pour le limon des Plateaux, formation d'origine éolienne qui peut être assez plastique. Il a été généralisé ces dernières années à des argiles ou des marnes de plus en plus grasses, lorsque des machines mixeuses plus performantes ont pu être utilisées, cependant que les bons remblais de ballastières devenaient de plus en plus chers, parce que plus rares.

L'ettringite, ou sel de Candlot, est un composant nécessitant la présence de chaux, de sulfate et d'aluminate. Il se forme naturellement dans la pâte du ciment durcie, mais peut se former aussi lorsque les aluminates du ciment rencontrent un sulfate. Il apparait alors - lentement - un sulfo-aluminate avec changement de forme cristalline, accompagné de dilatation. C'est le phénomène bien connu sous le nom générique d'alcali-réaction, qui apparaît comme le responsable du gonflement du sol et des désordres à Louvres, en 1985. MITCHELL (1986), dans une publication récente, a donné, lui aussi, cette interprétation à des désordres, de même nature, survenus aux U.S.A., après des traitements de sols à la chaux. D'après cet 
auteur, l'aluminate nécessaire à la formation de l'ettringite proviendrait des argiles elles-mêmes, dans le cas qu'il a étudié.

En l'absence d'une connaissance précise de la répartition du gypse dans le sol, il n'était évidemment pas possible de préciser pourquoi telle partie du dallage devait gonfler plus vite que telle autre. Mais, de toute façon, devant l'impossibilité d'éliminer la chaux excédentaire ou le gypse excédentaire, il n'y a rien à faire, sauf à enlever tout le remblai pour en reconstituer un autre, ou de suivre l'évolution du phénomène qui, par chance, était suffisamment régulier dans son extension en surface pour ne pas exiger de fréquentes réparations du dallage.

La fissuration de dallage et l'ouverture des joints faisaient craindre une expansion initiale horizontale des sols gonflants, ce qui, à la longue, aurait pu être dangereux pour les longrines et pour les têtes de pieux (fig. 1).

Des mesures de distance entre poteaux n'ont pas montré de mouvements bien convaincants jusqu'à présent, et il faut bien reconnaitre, d'une part, que les remblais extérieurs à la construction, probablement gonflants eux aussi, doivent exercer une certaine poussée compensatrice et, d'autre part, que l'expansion volumique, ayant une direction facile vers le haut, a do se faire préférentiellement en soulevant les dalles.

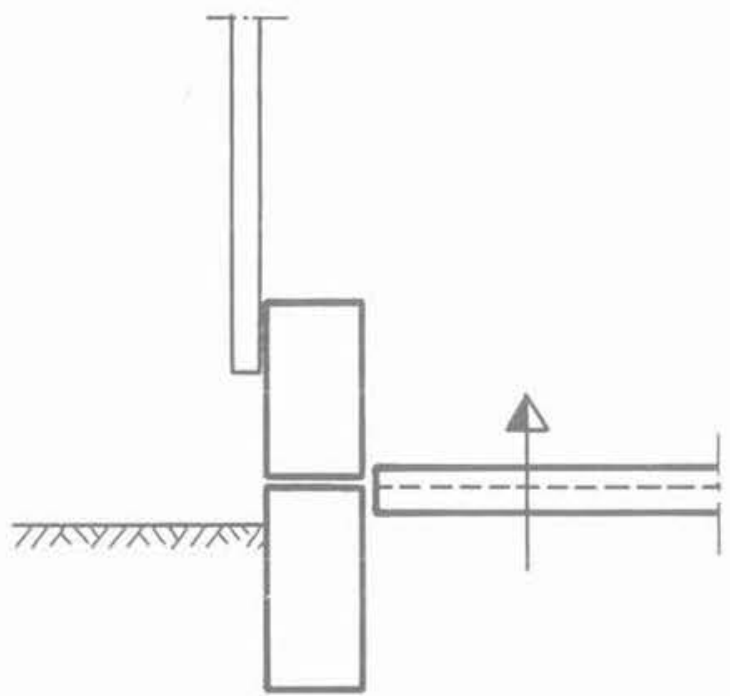

Fig. 3. - Principe de la réparation.

Fig. 3. - Repairs principle.

Devant de tels incidents, il est fondamental de ne pas effectuer les réparations trop vite pour ne pas prendre de risque de voir se rompre, à nouveau, ce qui vient d'être réparé car, malheureusement, les réactions chimiques de ce type sont très lentes; la figure 4 indique l'évolution des gonflements enregistrés pour un certain nombre de repères : il est bien clair qu'aucune déformation asymptotique n'est, pour le moment, en vue. Il faut donc se contenter de faire disparaître les causes de désordre, comme celles indiquées sur les figures 2 et 3 , grâce à des dispositions de réparation désolidarisant complètement les parties mobiles et les parties fixes, et par l'entretien des joints entre les dalles, de façon que le roulage des engins de manutention reste sans danger et que la continuité du service du bâtiment puisse être maintenue.

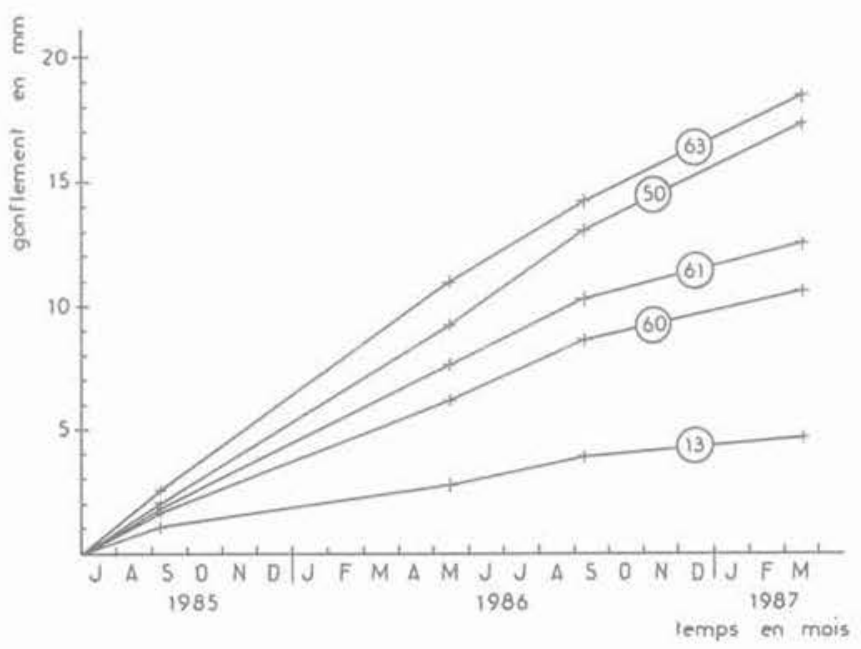

Fig. 4. - Gonflements en fonction du temps.

Fig. 4. - Swelling versus time.

\section{CONCLUSION}

L'exemple qui vient d'être décrit est tout à fait caractéristique d'un accident dont les conséquences sont d'autant plus fâcheuses que les gonflements différentiels sont grands. Il méritait d'être signalé car, si les sols contenant des sulfates sont relativement fréquents, la détection du gypse, par analyse chimique, est extrêmement simple et suffit à mettre en garde pour éviter d'employer en remblai et de traiter de tels matériaux à la chaux ou au ciment.

\section{BIBLIOGRAPHIE}

LACHAUD R. (1979), Thaumasite et ettringite dans les matériaux de construction. Annales I.T.B.T.P., mars 1979.

MITCHELL J.K. (1986), Practical Problems from Surprising Soil Behavior. 20 $0^{e}$ Terzaghi lecture, A.S.C.E. Journal of Geotechnical Engineering, vol. 112, n³, mars 1986 (Delayed Failure of Limestabilized Pavement Bases : pp. 274-279). 